\title{
SURREAL: Subgraph Robust Representation Learning
}

Saba A. Al-Sayouri ${ }^{1 *}$, Danai Koutra ${ }^{2 \dagger}$, Evangelos E. Papalexakis ${ }^{3+}$ and Sarah S. Lam ${ }^{1+}$

*Correspondence:
Ssyouri1@binghamton.edu
${ }^{+}$Danai Koutra, Evangelos E.
Papalexakis and Sarah S. Lam
contributed equally to this work.
'Systems Science and Industrial
Engineering Department,
Binghamton University, 4400 Vestal
Pkwy E, 13902 Binghamton, NY,
United States
Full list of author information is
available at the end of the article

\section{Abstract}

The success of graph embeddings or nodrepresentation learning in a variety of downstream tasks, such as node classification, link prediction, and recommendation systems, has led to their popularity in recent years. Representation learning algorithms aim to preserve local and global network structure by identifying node neighborhoods. However, many existing network representation learning methods generate embeddings that are still not effective enough, or lead to unstable representations due to random processes (e.g., random walks to generate context) and thus, cannot generalize to multi-graph problems. In this paper, we propose SURREAL, a novel, stable graph embedding algorithmic framework that leverages "spatio-electric" (SE) subgraphs: it learns graph representations using the analogy of graphs with electrical circuits. It preserves both local and global connectivity patterns, and addresses the issue of high-degree nodes that may incidentally connect a pair of nodes in a graph. Further, it exploits the strength of weak ties and meta-data that have been neglected by baselines. The experiments show that SURREAL outperforms state-of-the-art techniques by up to $37 \%$ ( $6 \%$ on average) on different multi-label classification problems. Further, in contrast to baseline methods, SURREAL, being deterministic, is stable and thus can generalize to single and multi-graph tasks.

Keywords: Large-scale information networks, Representation learning, Graph embeddings, Graph embeddings

\section{Introduction}

Conventional graph mining algorithms (Goyal and Ferrara 2017) have been designed to learn a set of hand-crafted features that best perform to conduct a specific downstream task; i.e., link prediction (Liben-Nowell and Kleinberg 2007), node classification (Bhagat et al. 2011), and recommendation (Yu et al. 2014). However, present research has steered the direction towards a more effective way to mine graphs: feature learning (Bengio et al. 2013). That is, a unified set of features that can effectively generalize over distinct graph mining-related tasks is exploited. To this end, recent research efforts have focused on designing either unsupervised or semi-supervised algorithms to learn node representations (Perozzi et al. 2014; Perozzi et al. 2016; Grover and Leskovec 2016; Tang et al. 2015). Such efforts have been initiated in the domain of natural language processing (NLP) (Mikolov et al. 2013; Le and Mikolov 2014; Mikolov et al. 2013), where two word2vec (Mikolov et al. 2013) models have been proposed, namely continuous bag of words (CBOW) and SkipGram. Inspired by the recent advancements in the NLP 
domain, and the analogy in the context, various algorithms have been developed to learn graph representations (Perozzi et al. 2014; Tang et al. 2015; Grover and Leskovec 2016). However, since real-world networks convey more complex relationships compared to those emerging in corpora, some recent representation learning algorithms (Perozzi et al. 2014; Perozzi et al. 2016; Grover and Leskovec 2016) generate representations that are still not effective enough in preserving network structure, and have room for improvement. This in turn impacts the quality of node representations, which compromises the performance of downstream processes. In addition, state-of-the-art algorithms, such as random walk-based methods, share a major stability issue that renders them less robust and applicable to multi-graph problems (Heimann and Koutra 2017; Heimann et al. 2018), such as graph similarity (Koutra et al. 2013) and network alignment (Bayati et al. 2009). This can be attributed to the fact that nodes on random walks are sampled by ID, where the basic assumption is that graphs are permuted and nodes that should align do not necessarily have the same ID. As a result, while baseline representation learning methods strive to preserve similarities among nodes in a single graph, they fail to maintain similarities across different runs of the methods, even with using the same dataset (Heimann et al. 2018) (graph similarity (Koutra et al. 2013) and network alignment (Bayati et al. 2009)) .

In addition to the representations' robustness, the quality of the learned representations is heavily influenced by the preserved local and global structure, matters. Therefore, we develop a robust graph embedding method that preserves connectivity patterns unique to undirected and (un)weighted graphs. It employs the concept of network flow represented by spatio-electric (SE) subgraphs. The SE subgraphs leverage the analogy with electrical circuits: a node is assumed to serve as a voltage source, and an edge is assumed to be a resistor, where its conductance is considered as the weight of the edge. Forming a SE subgraph allows to: (1) Concurrently capture the node local and global connections, (2) Account for the node degree imbalances by downweighing the importance of paths through high-degree nodes (hops), (3) Take into account both low- and high-weight edges; and (4) Account for meta-data that is largely being neglected by existing embedding methods. Meta-data represents network's flow that indicates the actual unnoticeable interactions that may take place between any two nodes in the network. Capturing network's flow can significantly improve that preservation of network global structure, in particular.

To summarize, our contributions are:

1. Flow-based Formulation. We propose a graph embedding approach that robustly preserves local and global structure by leveraging the notion of network flow to produce approximate but high-quality SE subgraphs between pairs of non-adjacent nodes in undirected and (un)weighted large-scale graphs. We use the formed SE subgraphs to identify the node neighborhoods and not restrict ourselves just to one- or two-hop neighbors.

2. Algorithm for Stable Representations. Contrary to all state-of-the-art methods, which involve randomness, reflected on the embeddings and their quality, our proposed SUbgraph Robust REpresentAtion Learning method, SURREAL ${ }^{1}$, produces consistent embeddings across independent runs.

3. Experiments. We extensively evaluate SURREAL empirically, and we demonstrate that it outperforms the state-of-the-art algorithms in two aspects. (1) Effectiveness: 
SURREAL outperforms state-of-the-art algorithms by up to $36.85 \%$ on multi-label classification problem, and (2) Robustness: in contrast to baseline methods, SURREAL is stable across different runs on the same dataset.

\section{Related work}

Representation Learning. Recent work in network representation learning has been largely motivated by the new progress in natural language processing (NLP) domain (Mikolov et al. 2013; Le and Mikolov 2014; Mikolov et al. 2013), due to the existing analogy among the two fields, where a network is represented as a document. One of the NLP leading advancements is rooted to the SkipGram model (Mikolov et al. 2013), due to its efficiency in scaling to large-scale networks. However, merely adopting the SkipGram model for graph representation learning seems to be insufficient in capturing local and global connections (Perozzi et al. 2014; Tang et al. 2015; Grover and Leskovec 2016), because of the sophisticated connectivity patterns emerge in realworld networks, but not in text corpora. Specifically, DeepWalk (Perozzi et al. 2014; Perozzi et al. 2016), for instance, employ small truncated random walks to approximate the neighborhood of a node in a graph. LINE (Tang et al. 2015) proposes to preserve the network local and global structure using first- and second-order proximities (Zhang et al. 2017; Goyal and Ferrara 2017), respectively. However, using the second-order proximity allows to preserve network structure only up to 2-hop neighbors. To the contrary, SURREAL is able to preserve more general global structure, since there is no pre-specified number of hops examined. Another approach, node2vec (Grover and Leskovec 2016), proposes to preserve homophily and structural equivalence using biased random walks. Recently, graph convolutional network (GCN) methods (Chen et al. 2018; Hamilton et al. 2017) are developed to learn network representations. Unlike these works, we propose to effectively and robustly learn node representations.

Proximity Subgraphs. There is a significant body of work addressing the problem of finding the relationships between a set of given nodes in a network. For instance, (Akoglu et al. 2013) finds simple pathways between a small set of marked nodes by leveraging the Minimum Description Length principle, while (Tong and Faloutsos 2006) defines the center-piece subgraph problem as finding the most central node with strong connections to a small set of input nodes. The work on connection subgraphs (Faloutsos et al. 2004), which capture proximity among any two non-adjacent nodes in arbitrary undirected and (un)weighted graphs, leverage the analogy with electrical circuits. Proximity subgraphs have also been employed for graph visualization (Rodrigues Jr. et al. 2006). Our work is the first to explore and extend connection subgraphs to define appropriate neighborhoods for representation learning.

\section{Problem formulation}

\section{Preliminary definition}

Information Network. An information network is defined as a graph $G=(\mathcal{V}, \mathcal{E})$, where $\mathcal{V}$ represents the set of nodes connected together by a set of edges $\mathcal{E}$. The weight of an edge $(u, v) \in \mathcal{E}, W_{u v}$, indicates the strength of the connection between nodes $u$ and $v$. In Table 1, we list the symbols and definitions used throughout the paper. 


\section{Problem definition}

Learning Large-scale Network Representations. Given a large-scale (un)weighted and undirected graph; $G=(\mathcal{V}, \mathcal{E})$, with $|\mathcal{V}|=n$ nodes and $|\mathcal{E}|=m$ edges, the problem of learning node representations seeks to preserve the network structure (e.g., local, global) while mapping each node $v \in \mathcal{V}$ from a high-dimensional feature space to a lowdimensional feature space $\mathbb{R}^{d}$ using a mapping function, $f_{G}: \mathcal{V} \rightarrow \mathbb{R}^{d}$, where $d \ll|\mathcal{V}|$. Since learning node representations aims to preserve network structure, state-of-the-art methods have employed various proximity metrics, such as first-order proximity (Goyal and Ferrara 2017; Zhang et al. 2017) that strives to preserve network local connections, and second-order proximity (Goyal and Ferrara 2017; Zhang et al. 2017) that aims to capture network global structure at a maximum distance of 2-hop neighbor from a given node.

Nevertheless, since a small percentage of a network's nodes are explicitly connected using first-order connections, and the large majority of network nodes are linked together via higher-order connections (e.g., second-order or higher), first- and second-order proximitiy are insufficient to effectively capture network structure, we propose to solve the following problem:

Given a (un)weighted and undirected graph $G(\mathcal{V}, \mathcal{E})$ with $n$ nodes and $m$ edges, and by using SE subgraphs,

Jointly Learn local and global network structure, and

Generate node representations.

We describe our proposed method, SURREAL, in detail next.

Table 1 Symbols and definitions

\begin{tabular}{|c|c|}
\hline Symbol & Definition \\
\hline$G=(\mathcal{V}, \mathcal{E})$ & (Un)weighted and undirected graph \\
\hline $\mathcal{V},|\mathcal{V}|=n$ & Set and number of vertices (nodes), respectively \\
\hline $\mathcal{E},|\mathcal{E}|=m$ & Set and number of links (edges), respectively \\
\hline$G^{\prime}$ & Modified graph after adding node $z$ \\
\hline $\mathcal{N}(u)$ & Set of direct neighbors of node $u$, i.e., nodes connected to $u$ \\
\hline$N_{E}(u)$ & Expanded neighborhood of node $u$ \\
\hline$N_{R}(u)$ & Refined neighborhood of node $u$ \\
\hline$E X$ & Set of expanded nodes that will form $N_{E}(u)$ (initialized to $\{u\}$ ) \\
\hline$P$ & Set of pending nodes, initialized to $u$ 's neighbors, $\mathcal{N}(u)$ \\
\hline$W_{u v}$ & Weight of an edge $(u, v) \in \mathcal{E}$ \\
\hline$V_{u}$ & Voltage of node $u$ \\
\hline $\operatorname{deg}(u)$ & Weighted degree of node $u$ \\
\hline$f_{G}$ & Mapping function \\
\hline$f(u)$ & Feature representation of node $u$ \\
\hline$D(u, v)$ & Distance between nodes $u$ and $v$ \\
\hline$C(v, w)$ & Weight/Conductance of the edge connecting nodes $v$ and $w$ \\
\hline$I(s, t)$ & Current flows between nodes $s$ and $t$ in the $N_{E}(u)$ \\
\hline $\operatorname{Pr}\left(N_{R}(u) \mid f(u)\right.$ & Probability of observing the refined neighborhood of $u$ given its feature representation \\
\hline$d$ & Dimensionality of learned representation \\
\hline$\alpha$ & Scalar; penalty parameter \\
\hline$M_{A}, X_{E} X_{R}$ & Desired size of $N_{E}(u)$ and $N_{R}(u)$, respectively \\
\hline$z$ & Universal sink node \\
\hline$c$ & Nearest common neighbor of non-neighboring nodes $u$ and $v$ \\
\hline$w$ & A node that belongs to $N_{R}(u)$ \\
\hline $\mathbb{R}$ & Set of real numbers \\
\hline NLP & Natural language processing \\
\hline CBOW & Continuous bag of words \\
\hline SE & Spatio-electric \\
\hline
\end{tabular}




\section{Proposed method: SURREAL}

In this section, we describe our proposed method, SURREAL, a deterministic algorithm that is capable of preserving local and global-beyond two hops-connectivity patterns. It consists of two main steps: (1) Neighborhood definition via SE subgraphs (3), which consists of neighborhood expansion and refinement phases, and (2) Node representation vector update (3). Since our method is deterministic, it can be applied to multi-graph problems, unlike previous works (Perozzi et al. 2014; Grover and Leskovec 2016; Perozzi et al. 2016) that employ random processes, such as random walks.

\section{Step 1: neighborhood definition}

The heart of learning node representations is to obtain representative node neighborhoods, which preserve local and global connections simultaneously. We propose to define node neighborhoods by leveraging the analogy between graphs and electrical circuits. Specifically, we introduce the idea of spatio-electric (SE) subgraphs, which are defined as proximity subgraphs that capture network structuer and formed on spatial and electrical bases. In particular, for a given node, we first expand its neighborhood on a distance basis to form the expanded subgraph. We afterward, refine the expanded subgraph on an electrical current (network flow) basis to ultimately generate the refined subgraph that represents the node neighborhood. We primarily summarize the benefit of using SE subgraphs in two points: (1) They render our method capable to better preserve network global structure-beyond two hops; and (2) Unlike baseline methods that employ random processes (e.g., random walks) for neighborhood generation, the use of SE subgraphs leads to generate robust neighborhoods, and thus robust representations across different runs on the same dataset.

The neighborhood definition step consists of two phases: (A) Neighborhood expansion, and (B) Neighborhood refinement. We describe each phase next, and give an illustration in Fig. 1.

\section{Phase A: neighborhood expansion - $N_{E}(u)$}

Given a node $u$, we propose to gradually expand its neighborhood on a distance basis. Specifically, we employ the analogy with electrical circuits in order to capture the distances between $u$ and the other nodes in the network, and then leverage these distances to guide its neighborhood expansion. This phase refers to the spatial part of the SE subgraphs formation process.

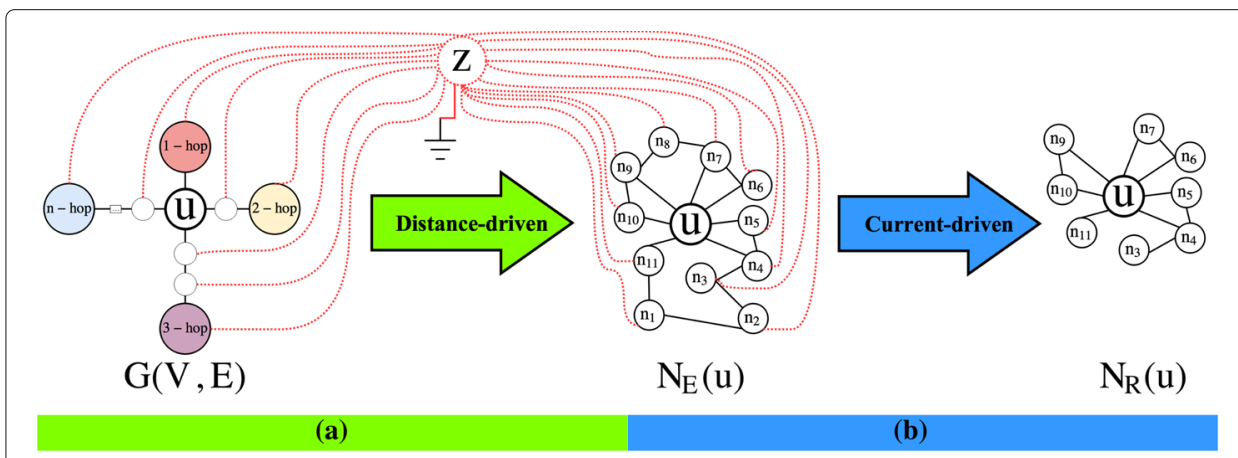

Fig. 1 A description of SURREAL algorithm neighborhood definition step main phases: (a) Neighborhood expansion of node $u$ through $n-$ hop neighbors to generate $N_{E}(u)$ on distance basis. Node $z$ indicates the grounded universal sink node. (b) Neighborhood refinement of $N_{E}(u)$ to generate $N_{R}(u)$ on current basis 
Graph Construction. We first construct a modified network $G^{\prime}$ from $G$ by introducing a universal sink node $z$ (grounded, with voltage $\left(V_{z}=0\right)$, and connect all the nodes (except from $u$ ) to that, as shown in Fig. 1a. The newly added edges in $G^{\prime}$ for every node $v \in\{\mathcal{V} \backslash u\}$ are weighted appropriately by the following weight or conductance (based on the circuit analogy):

$$
C(v, z)=\alpha \sum_{x \in \mathcal{N}(v) \backslash z} C(v, x),
$$

where $C(v, x)$ is the weight or conductance of the edge connecting nodes $v$ and $x, \mathcal{N}(v)$ is the set of 1-hop neighbors of $v$, and $\alpha>0$ is a scalar (set to 1 for unweighted graphs).

In the modified network $G^{\prime}$, the distance, or proximity, between the given node $u$ and every other node is defined as:

$$
D(u, v)= \begin{cases}\log \frac{\operatorname{deg}^{2}(u)}{C^{2}(u, v)}, & \text { for } v \in \mathcal{N}(u) \\ \log D(u, c)+D(c, v), & \text { for } v \notin \mathcal{N}(u), \text { and } u, v \in \mathcal{N}(c)\end{cases}
$$

where $\operatorname{deg}(u)$ is the weighted degree of $u$ (i.e., the sum of the weights of its incident edges), and the distance for non-neighboring nodes $u$ and $v$ is defined as the distance from each one to their nearest common neighbor $c \in \mathcal{V}$. During the neighborhood expansion phase, the distance formula addresses the issue of high-degree nodes (e.g., hops) that are generally incidentally connected-unrelated-to a specific node in the graph, but appear closer than low-degree nodes that may be truly connected-relatedto that node. Therefore, to downweigh the importance of paths through high-degree nodes, and serve our aim to include proximal nodes during the expansion phase, we significantly penalize the effect of high-degree nodes in the numerator using the term $C^{2}(u, v)$.

Distance-based Expansion. After constructing the circuit-based graph, we leverage it to expand $u$ 's neighborhood. Let $E X$ be the set of expanded nodes that will form the expansion graph $N_{E}(u)$ (initialized to $\{u\}$ ), and $P$ be the set of pending nodes, initialized to $u$ 's neighbors, $\mathcal{N}(u)$. During the expansion process, we choose the closest node to $u$ (except for $z$ ), as defined by the distance function in Eq. 2. Intuitively, the closer the expanded node $v$ to the source node $u$, the less information flow we lose. Once a node $v$ is added to the expansion subgraph, we add its immediate neighbors to $P$, and we repeat the process until we have $|E X|=M A X_{E}$ nodes, where $M A X_{E}$ is a user parameter that represents the desired size of expanded subgraph. We discuss the choice of $M A X_{E}$ in "Experiments" section. We show the neighborhood expansion pseudocode in Algorithm 1A. The procedure of computing the $N_{E}(u)$ takes $O(\mathcal{V})$ time. 


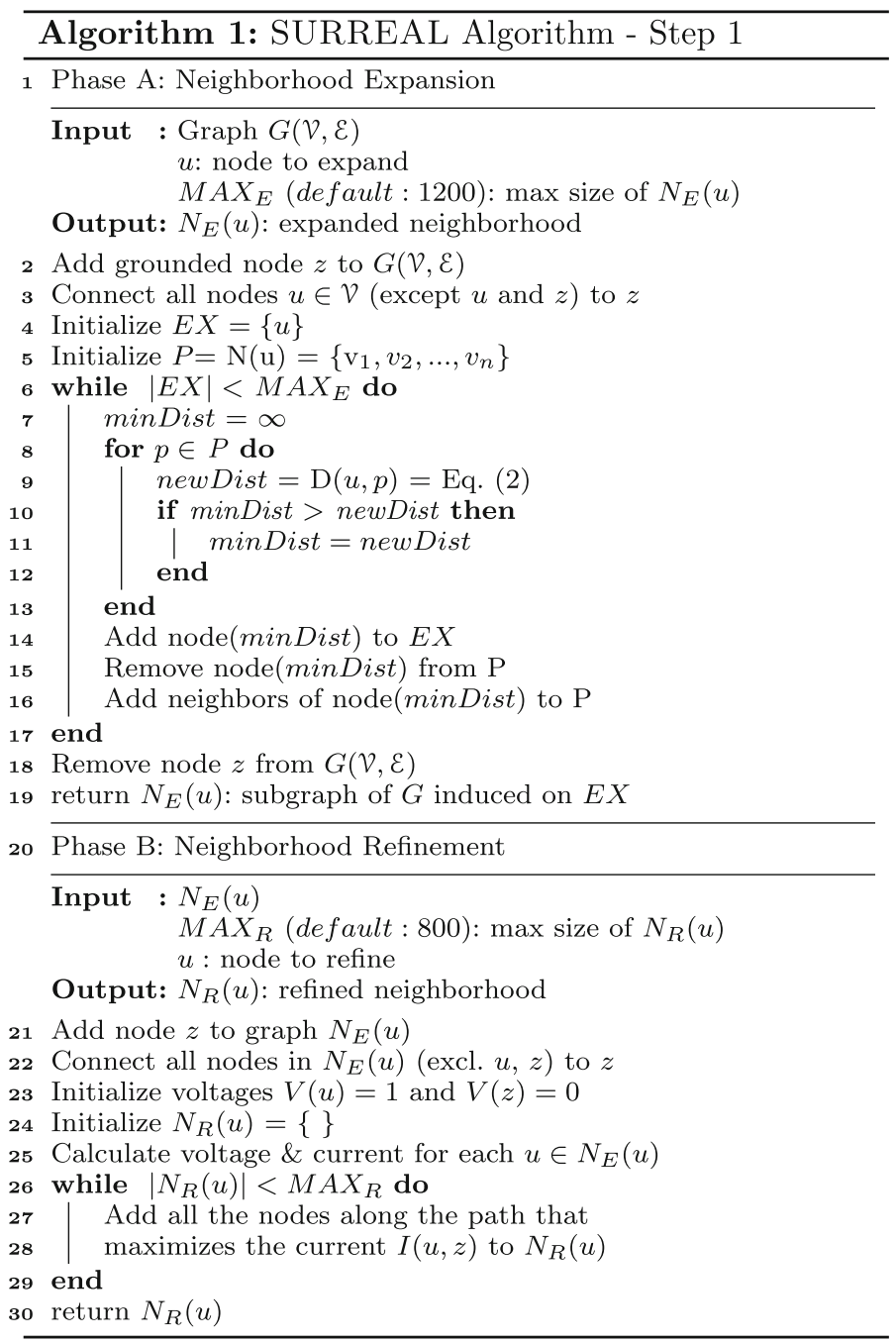

Example 1 Figure 2 shows one example of generating $N_{E}(u)$ for an undirected, unweighted graph $G$, in which the original edges have conductance (weight) equal to 1 , and the size of the expanded neighborhood is set to $M A X_{E}=5$. The conductances for the new edges in $G^{\prime}$ (red-dotted lines), computed via Eq.1, are shown in Fig. 2a. Based on the distances between $u$ and every other node, which are defined by Eq. 2 and shown in Fig. 2f, the neighborhood of $u$ is expanded on a distance basis.

\section{Phase B: neighborhood refinement $-N_{R}(u)$}

Since some nodes may appear close to the node we are expanding, and thus are included in its neighborhood during the expansion phase, while being barely interacted (low network flow), in this phase, we aim to refine the expanded neighborhood by removing existing nodes that are less related using a refinement process. As shown in Fig. 1b, the neighborhood refinement phase takes an expanded subgraph as an input and returns a refined neighborhood subgraph as an output, which is free of spurious graph regions. Unlike the previous phase that is based on distances, the refined subgraph is generated on a network flow (current) basis. Therefore, this phase relates to the electrical part of the SE subgraphs formation process. We show the neighborhood refinement pseudocode in Algorithm 1B. 


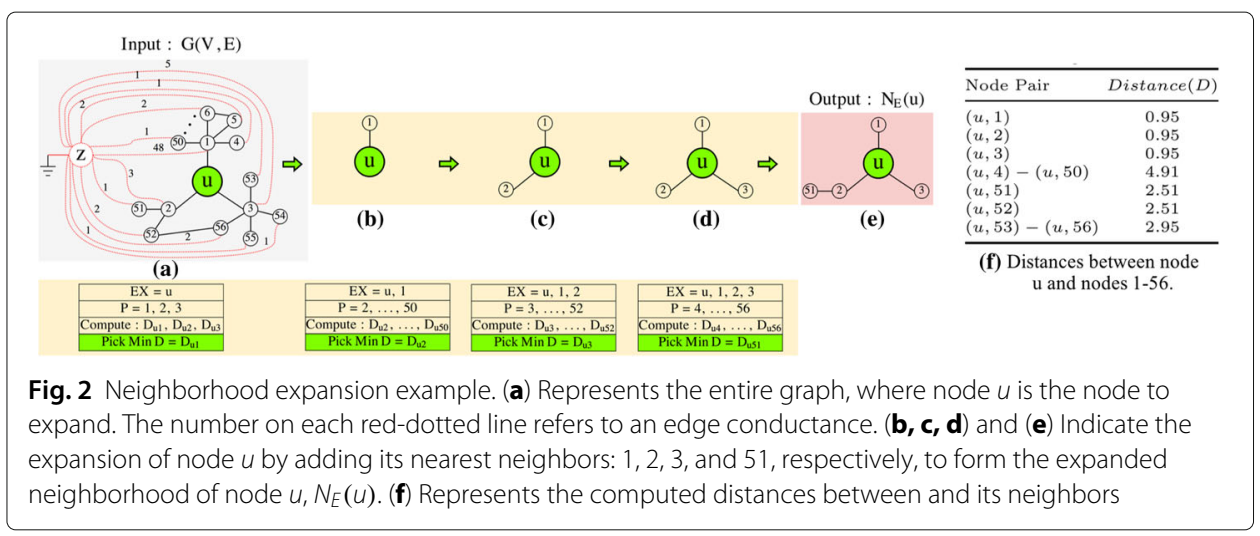

In a nutshell, in this phase, we first link the nodes of the expansion subgraph $N_{E}(u)$ (except for node $u$ ) to the previously introduced grounded node $z$. Then, we create the refined neighborhood subgraph by adding end-to-end paths from node $z$ to node $u$ one at a time, in decreasing order of total current. The underlying intuition of the refinement phase is to maximize the current that reaches to node $z$ from the source node $u$. By maximizing the current, we maximize the information flow between the source node $u$ and node $z$, which ultimately serves our goal of including proximal nodes to the source node $u$ in its $N_{R}(u)$. The process stops when the maximum predetermined refined subgraph size, $\left|N_{R}(u)\right|=M A X_{R}$, is reached. We discuss the choice of $M A X_{R}$ in "Experiments" section. Each time a path is added to the refined subgraph, only the nodes that are not already included in the subgraph are added. We use dynamic programming to implement our refinement process, which is like a depth first search (DFS) approach with a slight modification.

To that end, we need to calculate the current $I$ flows between any pair of neighbors in the expanded subgraph. In our context, $I$ indicates the meta-data or network flow. We compute the current $I$ flow from source node $s$ to target node $t$ using Ohm's law:

$$
I(s, t)=C(s, t) \cdot[V(s)-V(t)]
$$

where the $V(s)>V(t)$ are the voltages of $s$ and $t$, satisfying the downhill constraint (otherwise, there would be current flows in the opposite direction). In order to guarantee this satisfaction, we need to sort the subgraph's nodes in a descending order, based on their calculated voltage values, before we start current computations. The voltage of a node $s \in \mathcal{V}$ is defined as:

$$
V(s)= \begin{cases}\frac{\sum_{v \in \mathcal{N}(s)} V(v) \cdot C(s, v)}{\sum_{\nu} C(s, v)}, & \forall \text { nodes } s \neq u, z . \\ 1, & s=u . \\ 0, & s=z .\end{cases}
$$

where $C(s, v)$ is the conductance or weight of the edge between nodes $s$ and $v$, as defined in Eq. 1.

Example 2 Given the expanded neighborhood $N_{E}(u)$ in Example 1, the second phase of SURREAL gradually refines it on a current basis, as shown in Fig. 3. We first compute the voltages by solving the linear system in Eq. 4, and include them in the nodes of (b). Then, 


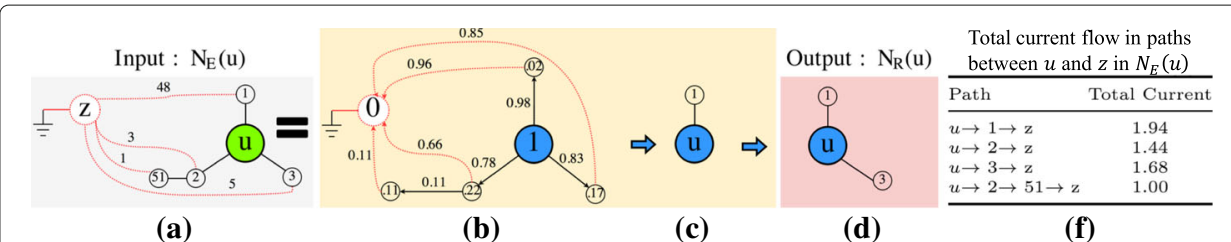

Fig. 3 Neighborhood refinement example. (a) Represents the expanded neighborhood of node $u, N_{E}(u)$. The number on each red-dotted line refers to an edge conductance. (b) Indicates the nodes' voltages (the numbers shown inside nodes) and currents flow through edges (reveal on the red-dotted lines) computations. (c) and (d) Indicate the refinement of node $u$ by adding the neighbors: node 1 and 3 that maximizes the current flows from node $u$ to node $z$ to form the refined neighborhood of node $u, N_{R}(u)$. (f) Refers to the computation of total current flows through each possible path between nodes $u$ and $z$

the current flow of each edge connecting nodes in the expanded neighborhood $N_{E}(u)$ is computed using Eq.3 such that the 'downhill constraint' is satisfied (current flowing from high to low voltage), as shown over the red-dotted edges in (b). Given the current values, we enumerate all possible paths between nodes $u$ and $z$, and give their total current flows in $(f)$. The paths are then added in descending order of total current into $N_{R}(u)$ until the stopping criterion is satisfied. In (c), we show the first such path. Finally, (d) gives the final neighborhood for $M A X_{R}=3$.

\section{Remarks}

(1) Need for SE Subgraphs. The notion of SE subgraphs is beneficial in our setting, since they allow us to: (1) Better control the search space, as we robustly generate node neighborhoods on distance and current bases without limiting ourselves to $K$-hop neighbors; (2) Benefit from the actual flow, meta-data, that is being neglected by state-of-the-art methods; (3) Exploit the strength of weak ties; (4) Avoid introducing randomness caused by random/biased walks; (5) Address the issue of high-degree nodes, that is, a node with a high-degree distribution has a low chance to be included in the SE subgraph, as a pair of nodes might be incidentally connected through a high-degree node; and (6) Better handle non-adjacent nodes that are ubiquitous in real-world large-scale graphs.

(2) SURREAL neighborhood vs. context in baseline methods. Unlike existing representation learning methods: (1) We preserve the local and global structure of network by accounting for the immediate neighbors and neighbors at increasing distances of the source node $u$ to identify its neighborhood; (2) We generate neighborhoods on distance and network flow bases; (3) We address the issue of high-node degree distribution; (4) We concurrently identify neighborhoods while maximizing proximity among non-adjacent nodes, which are abundant in real-world networks; and (5) We design our algorithm such that it yields consistent stable representations that suite single and multi-graph problems.

(3) SURREAL vs. connection subgraph algorithm (Faloutsos et al. 2004). It is important to note that the computations of "current" (in SURREAL) and "delivered current" (in (Faloutsos et al. 2004)) are different. The computation of current is not as informative as delivered current, but is more efficient. The use of delivered current was not a major struggle in (Faloutsos et al. 2004), because that algorithm only processes one subgraph. However, we find that it is problematic for generating multiple neighborhoods due to: (1) The large size of the expanded subgraph, $\left|N_{E}(u)\right|$; (2) The large size of refined subgraph, $\left|N_{R}(u)\right|$ (order of 800), compared to the display generation subgraph size capped at 30 
nodes; and (3) The extremely large number of subgraphs (equal to the number of nodes $|\mathcal{V}|=n)$ that need to be processed, to ultimately generate node neighborhoods.

(4) Computational complexity. The overall computational complexity of SURREAL is $O\left(\mathcal{V}^{2}\right)$

\section{Step 2: node representation vector update}

After identifying node neighborhoods in a graph, we aim to learn node representations via the standard SkipGram model (Mikolov et al. 2013). However, since SURREAL yields completely deterministic representations, we avoid the randomness implied by the SkipGram model by using the same random seed every time we employ it. The SkipGram objective maximizes the log-probability of observing the neighborhood generated during the neighborhood definition step, given each node's feature representation:

$$
\max _{f} \sum_{u \in V} \log \left(\operatorname{Pr}\left(N_{R}(u) \mid f(u)\right),\right.
$$

where $N_{R}(u)$ is the refined neighborhood of node $u$, and $f(u)$ is its feature representation. Following common practice, we make the maximum likelihood optimization tractable by making two assumptions:

Assumption 1 - Conditional independence. We assume that for node $u$, the likelihood of observing a neighboring node is completely independent of observing any other neighboring node, given node $u$ 's feature representation, $f(u)$ :

$$
\operatorname{Pr}\left(N_{R}(u) \mid f(u)\right)=\prod_{w \in N_{R}(u)} \operatorname{Pr}(w \mid f(u))
$$

where $w$ represents any node that belongs to node $u$ 's refined neighborhood.

Assumption 2 - Symmetry in feature space. The source node $u$ and any node $w$ in its refined neighborhood $N_{R}(u)$, have a symmetrical impact on each other in the continuous feature space. Therefore, the conditional probability, $\operatorname{Pr}(w \mid f(u))$, is modeled using the softmax function:

$$
\operatorname{Pr}(w \mid f(u))=\frac{\exp (f(w) \cdot f(u))}{\sum_{v \in V} \exp (f(v) \cdot f(u))}
$$

Based on the above two assumptions, we can simplify the objective in Eq. 5 as follows:

$$
\max _{f} \sum_{u \in V}\left[-\log \sum_{v \in V} \exp (f(v) \cdot f(u))+\sum_{w \in N_{R}(u)} f(w) \cdot f(u)\right]
$$

We note that performing such calculations for each node in large-scale graphs is computationally expensive. Therefore, we approximate the function using negative sampling (Mikolov et al. 2013). We optimize the objective shown in Eq. 8 using stochastic gradient descent.

\section{Experiments}

In this section, we aim to answer the following questions:

(Q1) How does SURREAL perform in multi-label classification compared to baseline representation learning approaches? (Q2) How stable are the representations that SURREAL and baseline methods learn? (Q3) How sensitive is SURREAL to its hyperparameters? 
Table 2 A brief description of evaluation datasets

\begin{tabular}{lllll}
\hline Dataset & \# Nodes & \# Edges & \# Labels & Network Type \\
\hline PPI (Grover and Leskovec 2016) & 3,890 & 76,584 & 50 & Biological \\
Wikipedia (Grover and Leskovec 2016) & 4,777 & 184,812 & 40 & Language \\
BlogCatalog (Grover and Leskovec 2016) & 10,312 & 333,983 & 39 & Social \\
CiteSeer (Tang et al. 2015) & 3,312 & 4,660 & 6 & Citation \\
Flickr (Mislove et al. 2007) & 80,513 & $5,899,882$ & 195 & Social \\
Youtube (Mislove et al. 2007) & $1,138,499$ & $2,990,443$ & 47 & Social \\
\hline
\end{tabular}

Before we answer these questions, we provide an overview of the datasets, and the baseline representation learning algorithms that we use in our evaluation.

Datasets. To showcase the generalization capability of SURREAL over distinct domains, we use a variety of datasets, which we briefly describe in Table 2.

Baseline Algorithms. We compare SURREAL with three state-of-the-art baselines: DeepWalk (Perozzi et al. 2014), node2vec (Grover and Leskovec 2016), and Walklets (Perozzi et al. 2016). The reason why we choose these state-of-the-art methods is the random way they adopt for neighborhood definition using random walks. On the contrary, in SURREAL, we follow a completely deterministic manner, which makes our method applicable for single and multi-graph problems. For all of the methods, we set the number of walks per node to 10 , walk length to 80 , the neighborhood size to 10 , and number of dimensions of the feature representation $d=128$. For node2vec, we set the return parameter $p=1$, and the in-out parameter $q=1$, in order to capture the homophily, and the structural equivalence connectivity patterns, respectively. For Walklets, we set the feature representation scale, $S=2$, which captures the relationships captured at scale 2 .

Experimental Setup. For SURREAL parameter settings, we set the expansion neighborhood subgraph size $\left|N_{E}(u)\right|=1200$. In order to compare with the baseline methods, we set the refinement neighborhood subgraph size, $\left|N_{R}(u)\right|=800$, and the number of dimensions of the feature representation, $d=128$, in line with the values used for DeepWalk, node2vec, and Walklets.

\section{Q1. Multi-label classification}

Setup. Multi-label classification is a single-graph canonical task, where each node in a graph is assigned a single or multiple labels from a finite set $\mathcal{L}$. We input the learned node representations to a one-vs-rest logistic regression classifier with L2 regularization. We repeat our experiments 10 times and report the mean Micro-F1 score results. We omit the

Table 3 Micro-F1 scores for multi-label classification on PPI, Wikipedia, and CiteSeer datasets

\begin{tabular}{llllllllll}
\hline Algorithm & \multicolumn{3}{c}{ PPI } & \multicolumn{3}{c}{ Wikipedia } & \multicolumn{3}{c}{ CiteSeer } \\
\cline { 2 - 11 } & $10 \%$ & $50 \%$ & $90 \%$ & $10 \%$ & $50 \%$ & $90 \%$ & $10 \%$ & $50 \%$ & $90 \%$ \\
\hline DeepWalk & 12.35 & 18.23 & 20.39 & 42.33 & 44.57 & 46.19 & 46.56 & 52.01 & 53.32 \\
node2vec & 16.19 & 20.64 & 21.75 & 44.38 & $\mathbf{4 8 . 3 7}$ & 48.85 & $\mathbf{5 0 . 9 2}$ & 52.49 & 56.72 \\
Walklets & 16.07 & 21.44 & 22.10 & 43.69 & 44.68 & 45.17 & 47.89 & 52.73 & 54.83 \\
SURREAL & $\mathbf{1 6 . 9 1}$ & $\mathbf{2 1 . 7 1}$ & $\mathbf{2 3 . 9 7}$ & $\mathbf{4 5 . 6 8}$ & 48.10 & $\mathbf{4 9 . 9 0}$ & 48.80 & $\mathbf{5 3 . 3 6}$ & $\mathbf{5 7 . 1 2}$ \\
\hline G.O. DWalk & 36.85 & 19.08 & 17.55 & 7.90 & 7.91 & 8.03 & 4.80 & 2.59 & 7.13 \\
G.O. N2vec & 4.41 & 5.16 & 10.19 & 2.92 & - & 2.14 & - & 1.63 & 0.70 \\
G.O. Walk & 5.19 & 1.23 & 8.47 & 4.53 & 7.64 & 10.48 & 1.87 & 1.18 & 4.17 \\
\hline Bolded numbers represent the best performance. By "G.O." we denote "gain over" & & & & &
\end{tabular}

Bolded numbers represent the best performance. By "G.O." we denote "gain over" 
Table 4 Micro-F1 scores for multi-label classification on BlogCatalog, Flickr, and Youtube datasets

\begin{tabular}{llllllllll}
\hline Algorithm & \multicolumn{3}{l}{ BlogCatalog } & \multicolumn{3}{c}{ Flickr } & \multicolumn{5}{c}{ Youtube } \\
\cline { 2 - 10 } & $10 \%$ & $50 \%$ & $90 \%$ & $10 \%$ & $50 \%$ & $90 \%$ & $10 \%$ & $50 \%$ & $90 \%$ \\
\hline DeepWalk & 30.12 & 34.28 & 34.83 & 37.70 & 39.62 & 42.36 & 40.62 & 42.09 & 43.77 \\
node2vec & $\mathbf{3 4 . 5 3}$ & $\mathbf{3 6 . 9 4}$ & $\mathbf{3 7 . 9 9}$ & 38.90 & 41.39 & 43.91 & 41.53 & 43.12 & 44.45 \\
Walklets & 26.90 & 29.09 & 30.41 & 38.32 & 40.58 & 42.62 & 41.15 & 42.97 & 43.89 \\
SURREAL & 31.02 & 34.85 & 36.42 & $\mathbf{3 8 . 9 8}$ & $\mathbf{4 2 . 3 1}$ & $\mathbf{4 4 . 2 6}$ & $\mathbf{4 1 . 9 4}$ & $\mathbf{4 3 . 7 6}$ & $\mathbf{4 5 . 2 6}$ \\
G.O. DWalk & 3.00 & 1.63 & 4.55 & 3.40 & 6.79 & 4.49 & 3.25 & 3.97 & 3.40 \\
G.O. N2vec & - & - & - & 0.21 & 2.22 & 0.80 & 0.10 & 1.48 & 1.82 \\
G.O. Walk & 15.27 & 19.80 & 19.75 & 1.72 & 4.26 & 3.85 & 1.92 & 1.84 & 3.12 \\
\hline
\end{tabular}

Bolded numbers represent the best performance. By "G.O." we denote "gain over"

results of other evaluation metrics-i.e., Macro-F1 score, because they follow the exact same trend. It is worth mentioning that multi-label classification is a challenging task, especially when the finite set of labels $\mathcal{L}$ is large, or the fraction of labeled vertices is small (Rossi et al. 2017).

Results. In Tables 3 and 4, we demonstrate the performance of SURREAL algorithm and compare it to the three representation learning state-of-the-art methods. Our results are statistically significant with a $p$-value $<0.02$. Overall, SURREAL outperforms or is competitive with the baseline methods, while also having the benefit of generalizing to the multi-network problems that the other methods fail to address. Below we discuss the experimental results by dataset.

PPI: It is remarkable that using various percentages of labeled nodes, SURREAL outperforms all the baselines. For instance, SURREAL is more effective than DeepWalk by $36.85 \%$ when the labeled nodes are sparse (10\%), $19.08 \%$ for $50 \%$ of labeled nodes, and $17.55 \%$ when the percentage of labeled nodes is $90 \%$.

Wikipedia: We observe that SURREAL outperforms the three baseline algorithms by up to $10.48 \%$ when using $90 \%$ of labeled nodes. In the only case where SURREAL does not beat node2vec, it is ranked second.

BlogCatalog: We observe that SURREAL has a comparable or better performance than DeepWalk and Walklets for various percentages of labeled nodes. Specifically, it outperforms DeepWalk by up to $4.55 \%$ and Walklets by up to $19.75 \%$, when the percentage of labeled nodes is $90 \%$. For more labeled nodes, SURREAL achieves similar performance to node2vec.

CiteSeer: Similar to Wikipedia, SURREAL outperforms the state-of-the-art algorithms, and achieves a maximum gain of $7.13 \%$ with $90 \%$ of labeled nodes.

Flickr: We perceive that SURREAL outperforms the other three baselines by up to $6.79 \%$, when using $50 \%$ of labeled nodes.

Youtube: We observe that SURREAL outperforms baseline methods, and achieves a maximum gain of $3.97 \%$ with $50 \%$ of labeled nodes.

Discussion: From the results, it is evident that SURREAL mostly outperforms the baseline techniques on PPI, Wikipedia, CiteSeer, Flickr, and Youtube networks, with exceptions, where SURREAL was very close to the best method. This can be rooted in the fact that SURREAL is more capable in preserving the global structure in such networks. On the other hand, although SURREAL has a very comparable performance with node2vec on BlogCatalog dataset, it might be that the $2^{\text {nd }}$ order biased random walks 
of node2vec are slightly more capable in preserving the homophily, and the structural equivalence connectivity patterns in BlogCatalog network.

\section{Q2. Representation learning stability}

Setup. Surveying the existing node representation learning methods, we perceive that the tasks for which such algorithms are being evaluated on are limited to single-graph related tasks-i.e., prediction, recommendation, node classification, and visualization. Since many tasks involve multiple networks (e.g., graph similarity (Koutra et al. 2013), graph alignment (Bayati et al. 2009), temporal graph anomaly detection (Koutra et al. 2013), brain network analysis for a group of subjects (Fallani et al. 2014)), we seek to examine the applicability of representations learning approaches to multi-network settings. Heimann and Koutra Heimann and Koutra (2017) states that existing embedding algorithms are inappropriate for multi-graph problems, and attributes this to the fact that different runs of any method yield different representations every time the technique is run even if the same dataset is used. To that end, SURREAL is fully deterministic, with the goal of achieving stable and robust outcomes. We evaluate SURREAL's stability by verifying the similarity of the learned vectors across different independent runs of the methods.

Results. Figure 4 shows the embeddings of two different runs of each approach against each other for a randomly selected set of nodes. For $d=128$, we visualize the results for three randomly selected dimensions of node2vec, DeepWalk, and Walklets. To avoid drawing a biased conclusion, we repeat the same experiment multiple times using different sets of randomly selected dimensions for the three baseline methods and the same observation holds. For SURREAL, we intentionally choose the same three dimensions randomly selected for each of the baseline methods. In the interest of space, we only show the visualization results of SURREAL using the same three dimensions $(39,55,111)$ used for Walklets method. The results are equivalent for all the dimensions. If all points fall on (or close to) the diagonal, this indicates stability, which is a desirable attribute of a robust graph embedding. Figure $4 \mathrm{a}-\mathrm{c}$ show that, as expected node2vec, DeepWalk, and Walklets, suffer from significant variation across runs. To the contrary, Fig. $4 \mathrm{~d}$ shows that SURREAL obtain perfectly consistent embeddings across runs, and thus it is robust.

\section{Q3. Parameter sensitivity}

For sensitivity analysis, we use the Wikipedia dataset with 50\% labeled nodes. We perform the following three experiments:

Size of the expansion neighborhood subgraph $\left|N_{E}(u)\right|$. First; we demonstrate the impact of varying the size of the expanded neighborhood, $\left|N_{E}(u)\right|$, in a multi-label classification problem. Therefore, we run SURREAL by varying the size of $N_{E}(u)$ from 600 to 1800 nodes in 200 increments. We limit the size of the refined neighborhood, $\left|N_{R}(u)\right|=$ 400. Figure 5a shows the Micro-F1 score results. We observe that by increasing the size of $N_{E}(u)$, the corresponding Micro-F1 score increases up to a certain limit $\left(\left|N_{E}(u)\right|=1000\right)$, while it starts to decrease afterwards. This can be attributed to the fact that enlarging the $N_{E}(u)$ to more than 1000 introduces noise to the generated neighborhood, which ultimately compromises the performance.

Size of the refinement neighborhood subgraph $\left|N_{R}(u)\right|$. Fixing the size of expanded neighborhood, $\left|N_{E}(u)\right|=1200$, we now examine the impact of altering the size of 

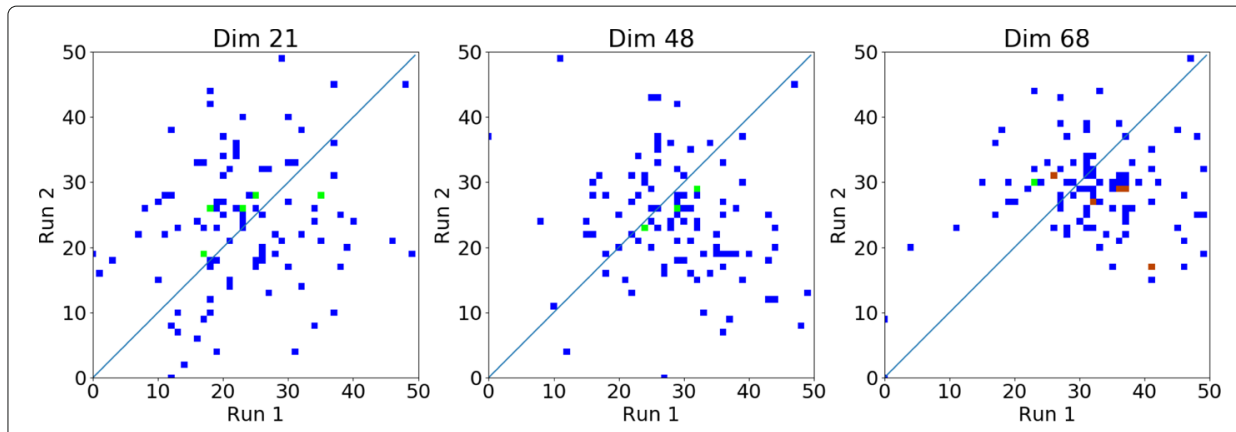

(a)
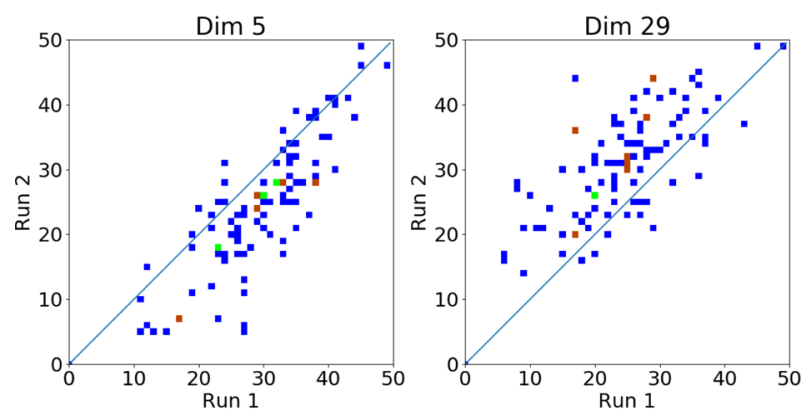

(b)
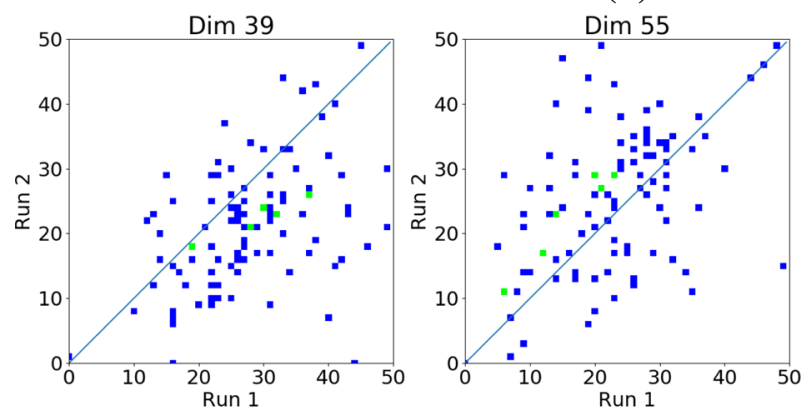

(c)
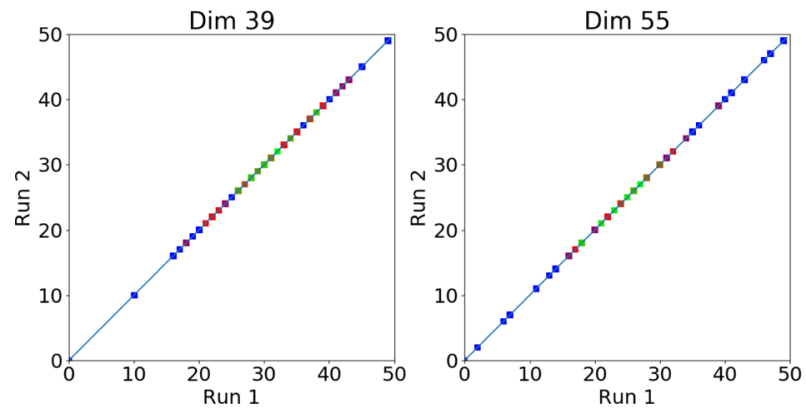

(d)
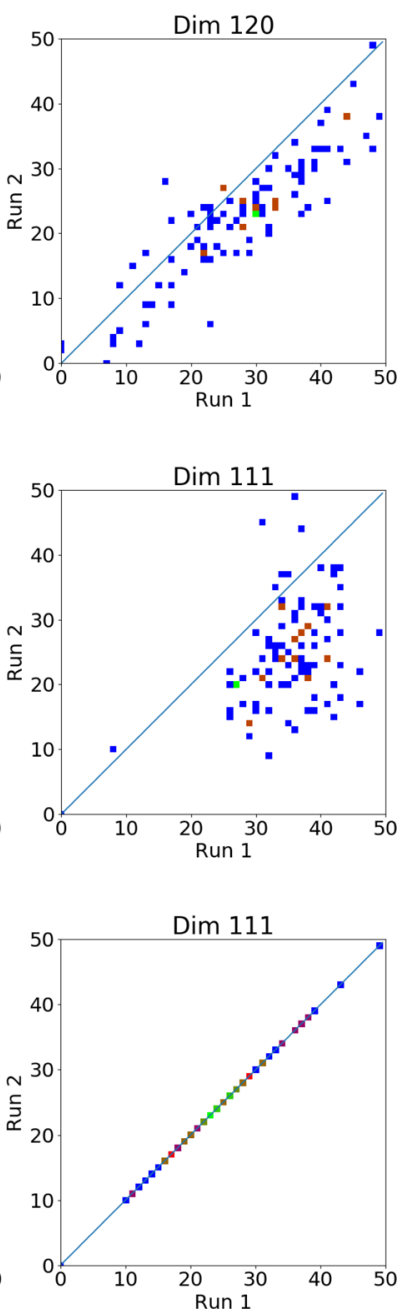

Fig. 4 PPI dataset: Comparison of embeddings per dimension for a random sample of 100 nodes. Node2vec, DeepWalk, Walklets, and SURREAL are run two times. The $x$-axis represents first run representations values, and the $y$-axis represents second run representations values. Three dimensions are selected randomly for each algorithm. The SURREAL-based representations are robust across runs (perfectly fall on a straight line $y=x$ ), which is not the case for node2vec, DeepWalk, and Walklets. The results are consistent for all the datasets. (a) Node2vec. Dimensions from left: 21, 48, 68 (b) DeepWalk. Dimensions from left: 5, 29, 120 (c) Walklets. Dimensions from left: 39, 55, 111 (d) SURREAL. Dimensions from left: 39, 55, 111 


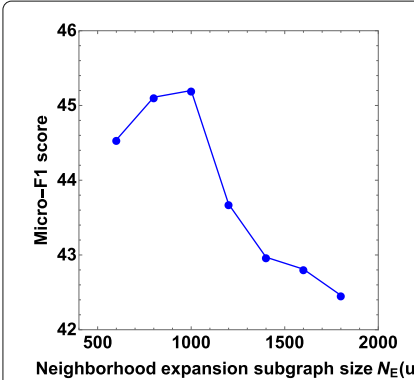

(a)

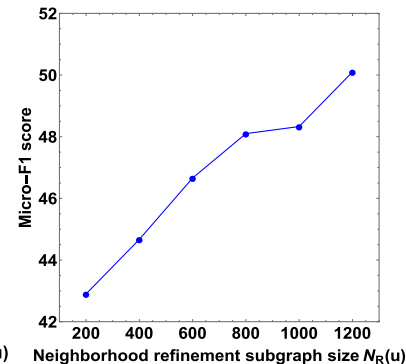

(b)

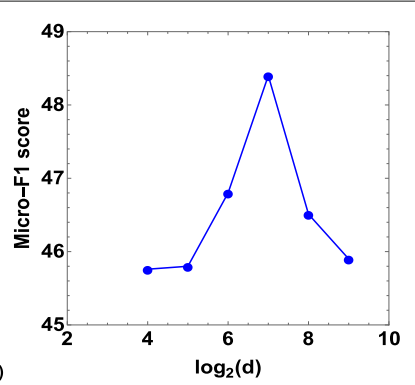

(c)

Fig. 5 Performance sensitivity analysis of SURREAL. (a) Expansion process for $\left|N_{E}(u)\right| \geq\left|N_{R}(u)\right|=400$. By increasing the size of $N_{E}(u)$, the corresponding Micro-F1 score decreases (b) Refinement process for $\left|N_{R}(u)\right| \leq\left|N_{E}(u)\right|=1200$. By increasing the size of $N_{R}(u)$, the corresponding Micro-F1 score increases (c) Vary $\log _{2}^{d}$ from 4-9. Micro-F1 score increases up to $\log _{2}^{d}=7$, and then it starts to decrease

the refined neighborhood, $\left|N_{R}(u)\right|$, in a multi-label classification problem. For that, we run SURREAL, while varying the size of $\left|N_{R}(u)\right|$ from 200 to 1200 nodes in 200 increments. Figure 5b shows the Micro-F1 results. We observe that increasing the $\left|N_{R}(u)\right|$ is accompanied by an increase in the Micro-F1 score. This is rooted in the fact that enlarging the $\left|N_{R}(u)\right|$ includes more useful information in the refined neighborhoods, which SkipGram model (Mikolov et al. 2013) leverages to learn and update the node representations.

Number of dimensions $\boldsymbol{d}$. Fixing the sizes of the expanded subgraph, $\left|N_{E}(u)\right|=1200$, and the refined subgraph, $\left|N_{R}(u)\right|=800$, we demonstrate the impact of varying the representation number of dimensions, $d$, in a multi-label classification problem. For that, we run SURREAL, while varying $\log _{2} d$ from 4 to 9 . Figure $5 \mathrm{c}$ shows the Micro-F1 results.

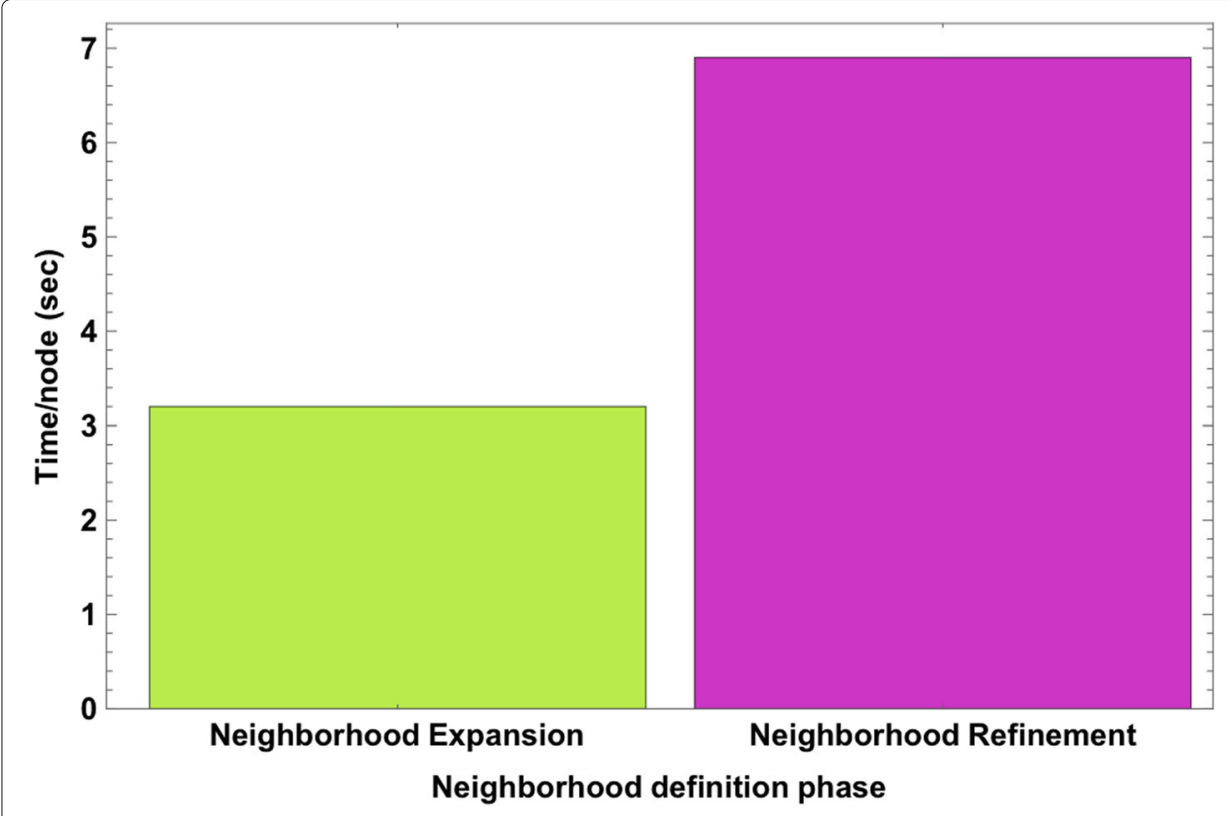

Fig. 6 Runtime analysis of SURREAL's neighborhood expansion and refinement phases, per node, of Wikipedia dataset. The $\left|N_{E}(u)\right|=1,200$ and $\left|N_{R}(u)=800\right|$. The refinement process represents a bottleneck that renders SURREAL less efficient 
We note that the Micro-F1 score constantly increases by increasing $\log _{2} d$ up to 7 , which corresponds to $d=128$, while it starts to drop afterwards. We root this in the fact that using higher number of dimensions could introduce unrelated dimensions to the representation space, which eventually impacts the performance.

\section{Runtime analysis}

To test for scalability, we run SURREAL's neighborhood definition step two phases: (1) Neighborhood expansion; and (2) Neighborhood refinement, and the corresponding runtime per node is reported (Fig. 6). For runtime analysis, we set the expanded subgraph size $\left|N_{E}(u)\right|=1,200$, the refinement subgraph size $\left|N_{R}(u)\right|=800$, and the representation dimensionality $d=128$. We observe that in order to expand and refine a single node, SURREAL takes 3.2 and 6.9 seconds, respectively. Apparently, the refinement neighborhood phase represents a bottleneck that renders SURREAL less efficient. Therefore, SURREAL is optimized as shown in "SURREAL's optimization" section.

\section{SURREAL's optimization}

In order to speedup SURREAL, the concept of graph summarization (Liu et al. 2018), where SURREAL can more efficiently handle large-scale graphs, is used. The abundance use of summarization concept is attributed to its capability of preserving and identifying the network structure and the meaning in data. Therefore, a compression-based summarization method (Stanley et al. 2018) is employed to summarize the networks utilized to evaluate the SURREAL method. This technique operates on a static and homogeneous-one entity and one link type-input graph. Further, it is defined as a grouping or aggregation-based method, because it employs a clustering technique to split a given graph $G$ into multiple clusters, which afterward, map each denselyconnected cluster into a supernode. The resultant summary graph is: (1) A supergraph that comprises supernodes and superedges connecting the supernodes together; (2) Flat, as the original nodes are simply grouped into several supernodes, on the same level of abstraction; and (3) Non-overlapping, where each node in the original graph is assigned to a single supernode in the summary graph. The problem of graph summarization is defined as follows:

Given a (un)weighted, undirected, static, and homogeneous graph $G(\mathcal{V}, \mathcal{E})$ with $n$ nodes and $m$ edges,

Find a summary graph: supergraph, to concisely depict the given graph.

The reasons why we choose (Stanley et al. 2018) as a graph summarization method are: (1) To decrease the runtime of SURREAL, especially for large-scale graphs; (2) Its ability to generate a small summary graph comparing to the original input large-scale graph, therefore, feeding the resultant summary graph into SURREAL would generate near-robust representations that resemble the ones generated using the original graph using SURREAL-the representations of each sueprnode and the nodes belong to it are identical;-(3) It accounts for network connectivity patterns while defining the supernodes that would ultimately serve as communities; (4) As this method is devoted for community detection purposes, the generated communities using the summarization method are consistent with the communities generated using the original network; (5) It is an unsupervised method, which aligns 


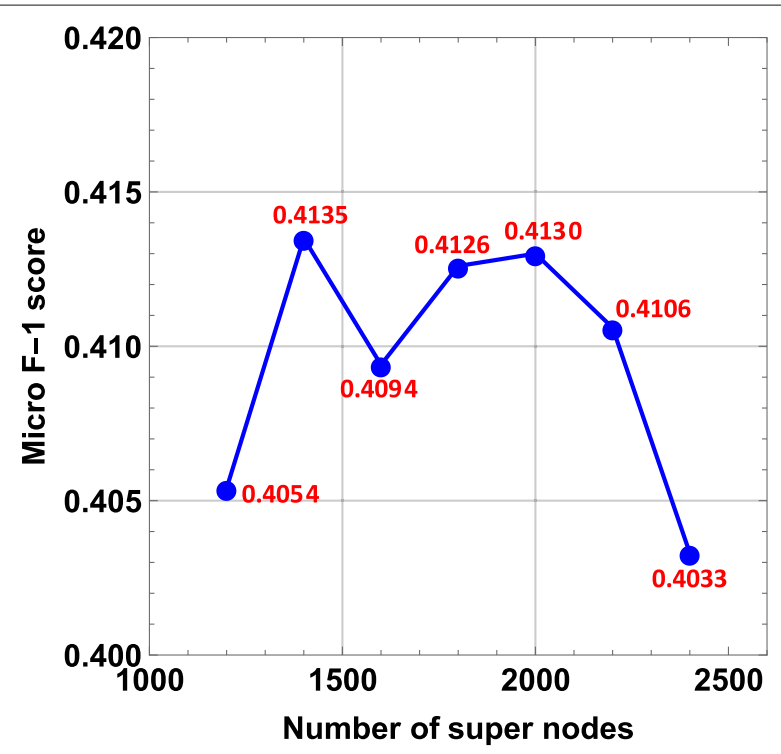

Fig. 7 SURREAL's Performance with respect to various numbers of supernodes of supergraph summary method for Wekipedia dataset. No significant growth or drop in performance while increasing the number of supernodes

with the robustness nature of SURREAL; and (6) It is categorized as a network pre-processing compression-based technique, where the entire set of nodes exists in the original graph is included in the summary graph, and agglomerated into supernodes.

As the number of supernodes is a user-defined parameter in (Stanley et al. 2018), we perform an experiment to report the SURREAL's performance with respect to different

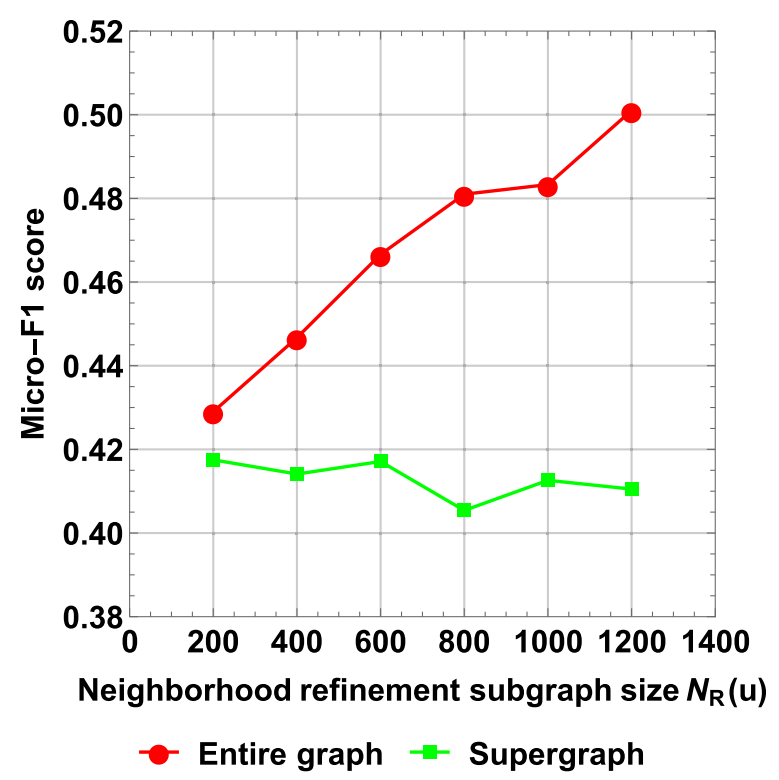

Fig. 8 Performance comparison of SURREAL using two input graphs: entire graph and supergraph. The experiment is conducted using Wikipedia dataset. The $\left|N_{R}(u)\right|$ is varied from 200 to 1,200, while

$\left|N_{E}(u)\right|=1,200$ and $d=128$. The performance degrades when using the supergraph instead of entire graph as an input graph 


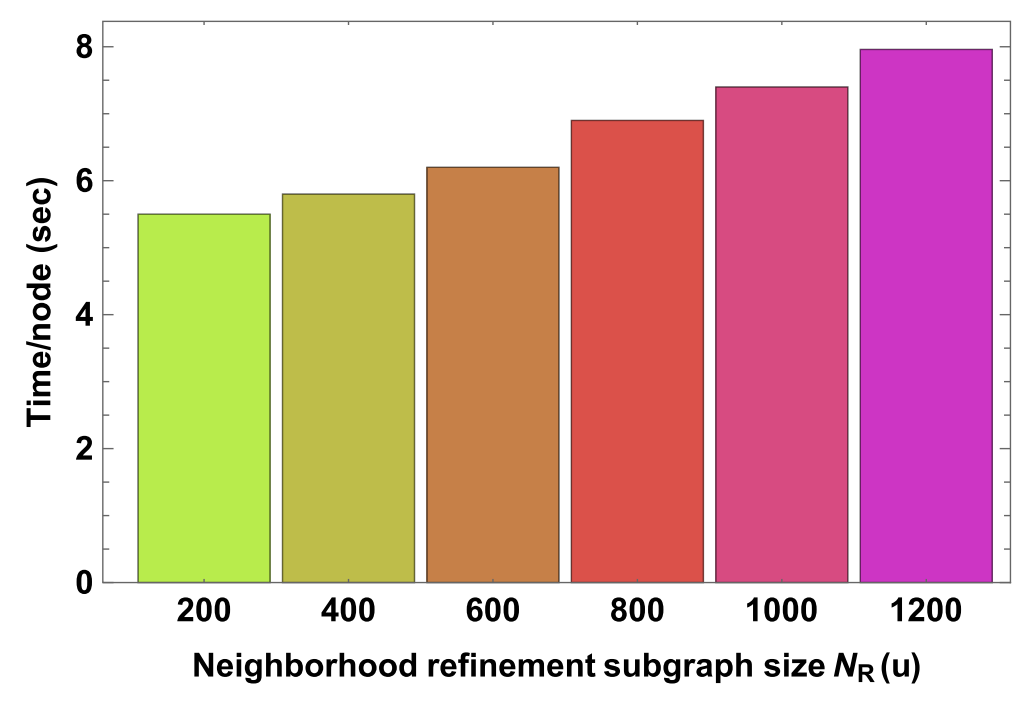

Fig. 9 Runtime analysis for different refinement subgraph sizes when using supergraph as an input graph to SURREAL. The $\left|N_{R}(u)\right|$ is varied from 200 to 1,200, while $\left|N_{E}(u)\right|$ and $d$ are set to 1,200 and 128, respectively

numbers of supernodes. The average Micro-F1 score is reported for Wikipedia dataset by altering the number of supernodes from 1200 to 2400 in 200 increments. Figure 7 shows that there is no significant increase or drop in performance when increasing the number of supernodes. Therefore, having 1,200 supernodes can optimize SURREAL, since setting the number of supernodes similar to the expanded subgraph size $\left|N_{E}(u)\right|$ will tremendously diminish the runtime of the expansion phase.

Due to the performance-efficiency trade-off, we perform an experiment using two input graphs: (1) The entire graph; and (2) The supergraph with 1,200 supernodes. We feed the input graph to SURREAL and report the average Micro-F1 score. We use Wikipedia dataset. We vary $\left|N_{R}(u)\right|$ from 200 to 1,200 , while setting $\left|N_{E}(u)\right|=1,200$ and $d=128$.

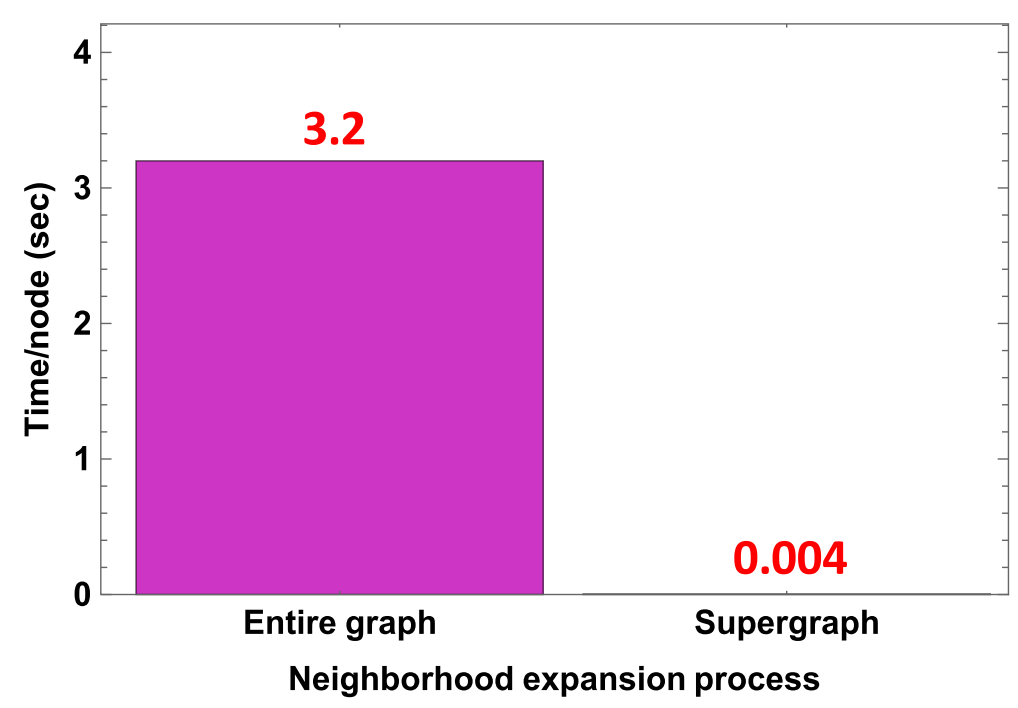

Fig. 10 Runtime analysis of neighborhood expansion process. SURREAL default settings are used: $\left|N_{E}(u)\right|=1,200,\left|N_{R}(u)\right|=800$, and $d=128$ 
Figure 8 shows that: (1) Increasing the $\left|N_{R}(u)\right|$ augments the difference in performance between the two input graphs, therefore, no significant difference is captured when using small values of $\left|N_{R}(u)\right|$; (2) There is a drop in performance when using a supergraph (green line) instead of the entire graph (red line) as an input graph, which confirms the performance-efficiency trade-off; and (3) The performance is more consistent when using the supergraph as an input graph.

As no significant change in performance captured when when using the supergraph and varying the refinement neighborhood subgraph size $\left|N_{R}(u)\right|$, we perform an experiment and report the runtime using supergraph as an input graph. We alter $\left|N_{R}(u)\right|$ from 200 to 1,200 , while setting $\left|N_{E}(u)\right|=1,200$ and $d=128$. Figure 9 shows that enlarging $\left|N_{R}(u)\right|$ is accompanied with a corresponding increase in runtime. Similar to observing the runtime of various refinement neighborhood subgraph sizes, we conduct another experiment to capture the expansion process runtime by setting $\left|N_{E}(u)\right|=1,200,\left|N_{R}(u)\right|=800$, and $d=128$. Figure 10 shows that when feeding the entire graph to SURREAL, it takes $3.2 \mathrm{~s}$ to expand a single node, while 0.0004 second when feeding a supergraph with 1,200 supernodes. That is, expanding a node that belongs to a supergraph is much faster than expanding a node in a full graph. Therefore, Figs. 9 and 10 confirm that although accompanied with a modest drop in performance, leveraging graph summarization concept greatly impacts SURREAL's efficiency.

\section{Conclusion}

We propose a novel and stable representation learning algorithm; SURREAL, using SE subgraphs. In contrast to representation learning baseline algorithms, SURREAL generates entirely deterministic representations, which makes it more appealing for single- and multi-graph problems. We empirically demonstrate SURREAL's efficacy and stability over state-of-the-art approaches. Experiments show that SURREAL is more or as effective as baselines, and is completely stable. As SURREAL is less scalable comparing to baseline techniques, we optimize SURREAL using the concept of graph summarization, where a compression-based method is employed. In our future work, we will address the interpretability aspect that is not well-examined in the representation learning literature. We will also address the issue of embedding update, especially for a recently-joined node that has no evident connections. This problem is very related to the "cold-start" problem in the recommendation systems, where a new user joins the system and we seek external information for this user, in order to properly compute his profile. Similarly, we will explore different forms of external context and meta-data for the recently-joined nodes, which can help us address connection sparsity.

\section{Acknowledgements}

Not applicable.

\section{Authors' contributions}

SA proposed, implemented, and evaluated the method. DK, EP, and SL improved the proposed method, suggested some of the evaluation experiments, and reviewed the manuscript. All authors read and approved the final manuscript.

Funding

Not applicable.

\section{Availability of data and materials}

The BlogCatalog, PPI, and Wikipedia datasets used for evaluation during the current study are available on SNAP, [https:// snap.stanford.edu/node2vec/\#datasets]. The CiteSeer dataset used for evaluation during the current study is available on GitHub, [https://github.com/thunlp/TADW]. The Flickr and Youtube datasets used for evaluation during the current study are available on Online Social Networks Research, [http://socialnetworks.mpi-sws.org/data-imc2007.html]. 


\section{Competing interests}

The authors declare that they have no competing interests.

\section{Author details}

'Systems Science and Industrial Engineering Department, Binghamton University, 4400 Vestal Pkwy E, 13902 Binghamton, NY, United States. ${ }^{2}$ Computer Science and Engineering Department, University of Michigan, 500 S State St, 48109 Ann Arbor, United States. ${ }^{3}$ Computer Science and Engineering Department, University of California, Riverside, 446 N Campus Dr, 92507 Riverside, United States.

Received: 13 March 2019 Accepted: 19 June 2019

Published online: 23 October 2019

\section{References}

Akoglu L, Chau DH, Vreeken J, Tatti N, Tong H, Faloutsos C (2013) Mining Connection Pathways for Marked Nodes in Large Graphs. In: Proceedings of the 2013 SIAM International Conference on Data Mining. Society for Industrial and Applied Mathematics, Austin. pp 37-45

Bayati M, Gerritsen M, Gleich DF, Saberi A, Wang Y (2009) Algorithms for large, sparse network alignment problems. In: Data Mining, 2009. ICDM'09. Ninth IEEE International Conference On. IEEE, Miami. pp 705-710

Bengio Y, Courville A, Vincent P (2013) Representation learning: A review and new perspectives. IEEE Trans. Pattern Anal. Mach Intell 35(8):1798-1828

Bhagat S, Cormode G, Muthukrishnan S (2011) Node classification in social networks. In: Social Network Data Analytics. Springer, Boston. pp 115-148

Chen J, Ma T, Xiao C (2018) Fastgcn: fast learning with graph convolutional networks via importance sampling. arXiv preprint. arXiv:1801.10247

Fallani FDV, Richiardi J, Chavez M, Achard S (2014) Graph analysis of functional brain networks: practical issues in translational neuroscience. Phil Trans R Soc B 369(1653):20130521

Faloutsos C, McCurley KS, Tomkins A (2004) Fast discovery of connection subgraphs. In: Proceedings of the Tenth ACM SIGKDD International Conference on Knowledge Discovery and Data Mining. ACM, Seattle. pp 118-127

Goyal P, Ferrara E (2017) Graph embedding techniques, applications, and performance: A survey. arXiv preprint. arXiv:1705.02801

Grover A, Leskovec J (2016) node2vec: Scalable feature learning for networks. In: Proceedings of the 22nd ACM SIGKDD International Conference on Knowledge Discovery and Data Mining. ACM, San Francisco. pp 855-864

Hamilton W, Ying Z, Leskovec J (2017) Inductive representation learning on large graphs. In: Advances in Neural Information Processing Systems. Neural Information Processing Systems, Long Beach. pp 1024-1034

Heimann M, Koutra D (2017) On generalizing neural node embedding methods to multi-network problems. In: ACM SIGKDD International Worshop on Mining and Learning with Graphs (MLG). ACM, Halifax, Nova Scotia

Heimann M, Shen H, Koutra D (2018) Node Representation Learning for Multiple Networks: The Case of Graph Alignment. ArXiv e-prints. 1802.06257

Koutra D, Vogelstein JT, Faloutsos C (2013) Deltacon: A principled massive-graph similarity function. In: Proceedings of the 2013 SIAM International Conference on Data Mining. SIAM, Austin. pp 162-170

Le Q, Mikolov T (2014) Distributed representations of sentences and documents. In: Proceedings of the 31st International Conference on Machine Learning (ICML-14). JMLR: W\&CP, Beijing. pp 1188-1196

Liben-Nowell D, Kleinberg J (2007) The link-prediction problem for social networks. J Assoc. Inf. Sci. Technol 58(7):1019-1031

Liu Y, Safavi T, Dighe A, Koutra D (2018) Graph summarization methods and applications: A survey. ACM Comput Surv (CSUR) 51(3):62

Mikolov T, Chen K, Corrado G, Dean J (2013) Efficient estimation of word representations in vector space. arXiv preprint. arXiv:1301.3781

Mikolov T, Sutskever I, Chen K, Corrado GS, Dean J (2013) Distributed representations of words and phrases and their compositionality. In: Advances in Neural Information Processing Systems. pp 3111-3119

Mislove A, Marcon M, Gummadi KP, Druschel P, Bhattacharjee B (2007) Measurement and Analysis of Online Social Networks. In: Proceedings of the 5th ACM/Usenix Internet Measurement Conference (IMC'07), San Diego

Perozzi B, Al-Rfou R, Skiena S (2014) Deepwalk: Online learning of social representations. In: Proceedings of the 20th ACM SIGKDD International Conference on Knowledge Discovery and Data Mining. ACM. pp 701-710

Perozzi B, Kulkarni V, Skiena S (2016) Walklets: Multiscale graph embeddings for interpretable network classification. arXiv preprint. arXiv:1605.02115. New York

Rodrigues Jr. JF, Tong H, Traina AJM, Faloutsos C, Leskovec J (2006) Gmine: A system for scalable, interactive graph visualization and mining. In: Proceedings of the $32 \mathrm{Nd}$ International Conference on Very Large Data Bases. VLDB '06. VLDB Endowment, Seoul. pp 1195-1198

Rossi RA, Zhou R, Ahmed NK (2017) Deep feature learning for graphs. arXiv preprint. arXiv:1704.08829

Stanley N, Kwitt R, Niethammer M, Mucha PJ (2018) Compressing networks with super nodes. Sci Rep 8(1):10892

Tang J, Qu M, Wang M, Zhang M, Yan J, Mei Q (2015) Line: Large-scale information network embedding. In: Proceedings of the 24th International Conference on World Wide Web. ACM, Florence. pp 1067-1077

Tong H, Faloutsos C (2006) Center-piece subgraphs: Problem definition and fast solutions. In: Proceedings of the 12th ACM SIGKDD International Conference on Knowledge Discovery and Data Mining. ACM, Philadelphia. pp 404-413

Yu X, Ren X, Sun Y, Gu Q, Sturt B, Khandelwal U, Norick B, Han J (2014) Personalized entity recommendation: A heterogeneous information network approach. In: Proceedings of the 7th ACM International Conference on Web Search and Data Mining. ACM, New York. pp 283-292

Zhang D, Yin J, Zhu X, Zhang C (2017) Network representation learning: A survey. arXiv preprint. arXiv:1801.05852

\section{Publisher's Note}

Springer Nature remains neutral with regard to jurisdictional claims in published maps and institutional affiliations. 